\title{
REVIEW
}

\section{The Future of Dry Eye Treatment: A Glance into the Therapeutic Pipeline}

\author{
Laura A. Vickers · Preeya K. Gupta
}

To view enhanced content go to www.ophthalmology-open.com

Received: July 6, 2015 / Published online: August 20, 2015

(C) The Author(s) 2015. This article is published with open access at Springerlink.com

\section{ABSTRACT}

Dry eye syndrome is both a primary disease and a secondary result of many pathological states of the eye. The symptoms range from mild to severe itching, burning, irritation, eye fatigue, and even vision loss that can lead to disability. Dry eye affects approximately 60 million people worldwide; as a result, medications to treat dry eye comprise approximately $15 \%$ of the ophthalmic pharmaceutical market. While doctors and patients eagerly await new treatments, pharmaceuticals in the pipeline are moving through the approval process with several promising drugs having completed phase 3 clinical testing. This review summarizes the findings of studies of the most promising, upcoming dry eye treatments in phase 2 and 3 clinical trials in the USA.

Keywords: Blepharitis; Dry eye; Human serum albumin; Integrin; Iontophoresis; LFA-1; Mucus penetrating particle; Peptidomimetic; Punctal plug; Serum tears

L. A. Vickers · P. K. Gupta (ه)

Department of Ophthalmology, Duke University

Eye Center, Durham, NC, USA

e-mail: preeya.gupta@duke.edu

\section{INTRODUCTION}

Dry eye disease (DED) affects millions of people around the world [1]. Whether due to aqueous deficiency or evaporative dry eye (or most commonly a combination of factors), it is a potentially debilitating condition. Functional limitation is variable, but moderate to severe DED may make it difficult for patients to do computer work, reading, and other activities that limit their daily lives and productivity. DED was traditionally thought to be a disease of age and more common in females. In recent years, a younger population is the most rapidly growing segment of dry eye sufferers, likely in part to shifts in our lifestyles toward frequent computer and visual display tasking. Post-refractive surgery (e.g., post-LASIK) dry eye is also a significant clinical obstacle [2]. The current mainstay treatment for mild dry eye includes various types of lubricating drops and ointments. These must be frequently re-applied and do not resolve the underlying disease process, often only temporarily relieving the symptoms.

This article is based on previously conducted studies and does not involve any new studies of 
human or animal subjects performed by any of the authors.

\section{Dry Eye as an Inflammatory State- Current Treatments and Implications for Pharmaceutical Development}

Dry eye has been recognized and treated as an inflammatory process. The first pharmaceutical to target an inflammatory pathway in order to treat dry eye is cyclosporine $0.05 \%$ (Restasis, Allergan; Irvine, CA, USA), which has become a mainstay of treatment for moderate to severe dry eye disease. While many patients benefit from this drug, some also have mixed reactions and may discontinue it over time due to intolerance of associated side effects (temporary redness and/or burning upon instillation) [3]. The need for additional treatments for dry eye is clear to patients and physicians alike, and the recognition of dry eye as an inflammatory process has opened the door to pharmaceuticals targeting the inflammatory cascade. Fortunately for dry eye patients, there are numerous promising pharmaceuticals in the pipeline, some borrowing insight from treatment of inflammatory conditions in other parts of the body, and many of them having completed or currently undergoing phase 3 clinical testing. Additionally, dry eye has become a target for device development, including for example the Oculeve Neurostimulator device and the EyeGate Ocular Iontophoresis device. These devices address the treatment of dry eye with very different and innovative approaches, which are reviewed below. Clinical trials for medications and devices to treat DED need to be well-designed in order to be successful, as dry eye is a disease in which symptoms may not correlate well with objective testing [1]. Even prior large-scale studies of DED have varied significantly in their method of assessing disease severity [4-6]. Despite this challenge, using a combination of subjective patient symptom questionnaires and objective outcome measures, several promising developments are on the horizon. This review summarizes the major developments for dry eye treatment currently in the therapeutic pipeline.

\section{PHARMACEUTICALS IN PHASE 3 CLINICAL TRIALS}

\section{Lifitegrast 5\%: Small-Molecule Integrin Antagonist}

Lifitegrast (Shire Pharmaceuticals; Lexington, MA, USA) is a small-molecule antagonist of the $\mathrm{T}$ cell integrin, lymphocyte function antigen-1 (LFA-1) [7-9]. LFA-1 plays a role in the inflammatory cascade of dry eye, binding intercellular adhesion molecule-1 (ICAM-1) and promoting T-cell activation and eventually cytokine release. Therefore, inhibiting LFA-1 effectively limits T-cell mediated inflammation. OPUS-1, a phase 3, randomized, prospective, double-masked placebo-controlled study compared lifitegrast twice daily with placebo in 565 subjects with a history of bilateral dry eye disease over the course of 12 weeks [7]. Measured outcomes included corneal fluorescein staining and conjunctival lissamine staining, Schirmer tear test, visual analog scale, and ocular discomfort score. Patients receiving lifitegrast showed statistically significant superior improvement over placebo in total corneal staining, total lissamine staining, mean eye dryness score, and ocular discomfort score over the course of the study, while other measured outcomes were not statistically different between the treatment and placebo groups at 12 weeks. Decreased lissamine 
staining in the treatment group was demonstrated as early as day 14, which may indicate a relatively rapid decrease in ocular surface inflammation with lifitegrast. Some limitations of this phase 3 study include the relatively short follow-up period. OPUS-2, completed in 2013, again compared lifitegrast twice daily with placebo for 12 weeks in dry eye patients with a history of active artificial tear use [10]. The primary endpoint of patient-reported symptoms was significantly better versus placebo, whereas there was no significant difference among the treatment and placebo groups in inferior corneal fluorescein staining score. OPUS-3, a third phase 3 trial, is in progress to further evaluate the efficacy and safety of lifitegrast. SONATA was a 1-year prospective study of lifitegrast versus placebo in 331 patients, with the goal to evaluate safety of long-term treatment [11]. The protocol for this study, completed in 2014, also allowed for use of concomitant artificial tears and other adjunctive topical treatments. Overall the drug was found to be well tolerated, but of note, almost $50 \%$ of patients receiving lifitegrast experienced some mild treatment-related side effect, most commonly dysgeusia and instillation site irritation. There were no vision-threatening side effects. Future studies may investigate the potential role for lifitegrast in patients with blepharitis and other diseases of ocular surface inflammation. Overall, lifitegrast shows promise in improving dry eye and is pending review by the FDA for approval in late 2015.

\section{Rebamipide: Quinolinone Derivative Mucin Secretogogue}

Rebamipide (Otsuka Pharmaceutical Co.; Tokyo, Japan) is a mucin secretogogue initially marketed in Japan for the treatment of gastric mucosal disorders and gastritis [12-16]. As decreased numbers of goblet cells have been observed in the conjunctiva and decreased mucin at the corneal surface has been observed in patients with dry eye, rebamipide was thus a potential target for dry eye treatment by stabilizing the mucin component of the tear film. Early studies demonstrated an increased production of periodic acid-Schiff-positive cells (goblet cells) when rebamipide was instilled into rabbit eyes and increased mucin-like glycoprotein when rebamipide was incubated with human corneal epithelial cells [17, 18]. Recent phase 2 and phase 3 studies have yielded promising results for clinical use of rebamipide in the treatment of dry eye $[13,16]$. In the phase 3 trial, 188 patients with dry eye syndrome were randomized to $2 \%$ rebamipide four times daily or $0.1 \%$ sodium hyaluronate six times daily for 4 weeks. The main outcome measures included two primary end points of corneal fluorescein staining score and lissamine green conjunctival staining score, in addition to Schirmer's test, tear film breakup time, subjective report of foreign body sensation, dryness, pain and blurred vision, and patient's treatment impression scores. At 4 weeks, mean change from baseline in corneal fluorescein staining scores verified non-inferiority to sodium hyaluronate. Lissamine conjunctival staining scores demonstrated superiority of rebamipide. While there was not a significant difference between the two groups in Schirmer's test and tear breakup time, foreign body sensation and eye pain scores were significantly superior in the rebamipide group, with improved efficacy noted at the 2 week time point. Of note, patients' overall impression of the improvement in symptoms was significantly better in the rebamipide group as well. Adverse events were rare, and included bitter taste, eye pruritis, nasopharyngitis, headache, and 
decrease in white cell count (which was noted in three patients in the rebamipide group and none in the sodium hyaluronate group). The decrease in white blood cell count was not further characterized in this study, and the implications of this finding may require further investigation. Additionally, the short duration of this phase 3 study, only 4 weeks, means that further work will be needed to demonstrate the potential role of rebamipide in the treatment of chronic dry eye syndrome. This medication was launched in Japan for the treatment of dry eye in a 2\% ophthalmic suspension in January 2012 and has yet to be approved for use in the USA.

\section{MiM-D3: Nerve Growth Factor Peptidomimetic, Mucin Secretogogue}

MIM-D3 (Mimetogen Pharmaceuticals; Gloucester, MA, USA) is a first in its class, small-molecule nerve growth factor (NGF) peptidomimetic that completed a phase 3 clinical trial for the treatment of dry eye in 2014 [19-22]. NGF plays a role in corneal wound healing and has previously been shown to have mucin secretogogue activity in conjunctival cells. Early studies in a rat model with scopolamine-induced dry eye demonstrated a favorable effect on glycoconjugate secretion with topical MIM-D3 $1 \%$ [20]. A subsequent phase 2 clinical trial enrolled 150 patients with dry eye who were randomized to $1 \%$ MIM-D3, 5\% MIM-D3 or placebo, dosed twice daily for 28 days [19]. This study, as well as the phase 3 trial, used the Controlled Adverse Environment (CAE) challenge to measure dry eye severity, where patients are subjected to an environment that exacerbates dry eye symptoms. Compared to placebo, fluorescein corneal staining post-CAE after 28 days of treatment with MIM-D3 was significantly improved in the 1\% MIM-D3 group. Patients in the 5\% MIM-D3 group showed better daily ocular dryness scores, and patients with higher symptom scores reported improvement of symptoms for both MIM-D3 doses. Adverse events were rare, including eye irritation and dellen formation, and systemic adverse events were not thought to be attributable to treatment. Limitations of the phase 2 study include the multiple post hoc analyses and short follow-up period. The subsequent phase 3 trial enrolled 403 patients with dry eye who received MIM-D3 $1 \%$ or placebo twice daily for 28 days. Primary outcome measures of corneal fluorescein staining and ocular dryness as well as several secondary outcomes were examined, again in response to the CAE [23]. The phase 3 study has not been published as yet; however, initial top-line results from Mimetogen demonstrated superiority over placebo in total corneal fluorescein staining. Notably, the mean blurred vision, reading, and TV-watching scores were improved with MIM-D3 compared with placebo [23]. Adverse events were described as rare and transient.

\section{OTX-DP: Sustained Release \\ Dexamethasone Loaded Punctal Plug \\ $0.4 \mathrm{mg}$}

In 2015, Ocular Therapeutix (Bedford, MA, USA) announced the outcomes of their second phase 3 clinical trial for OTX-DP, the sustained release dexamethasone-loaded punctal plug [24]. The punctal plug dispenses a tapered release of dexamethasone onto the ocular surface over the course of (up to) 30 days after insertion. OTX-DP has primarily been investigated for treatment of inflammation and pain in the postoperative period after 
cataract surgery. An initial phase 2 trial demonstrated superiority over placebo for absence of anterior chamber cells at days 14 and 30 and for absence of pain at all measured time points through 30 days after cataract surgery [25]. The plug was retained in all patients through day 14 and in about $97 \%$ of patients at 30 days. Importantly, the OTX-DP was also reported to be well tolerated by patients, and there were no significant elevations in intraocular pressure. The first phase 3 trial enrolled 247 patients undergoing cataract surgery in the US who were randomized to placement of the OTX-DP or standard, non-medicated vehicle punctal plug at the end of the surgical procedure. Outcome measures included anterior chamber inflammation and pain in the postoperative period. Patient-reported pain at day 8 and anterior chamber inflammation at day 14 after surgery were both statistically significantly better in the OTX-DP group versus placebo. In a second phase 3 trial enrolling 240 patients, however, a statistically significant decrease in patient-reported pain at day 8 after surgery was found in the OTX-DP group, but no significant difference in anterior chamber inflammation was detected at day 14. Further post hoc analysis is underway to investigate the differences in outcomes of these two trials. Potential advantages of OTX-DP include more reliable dosing of steroid and fewer self-administered medications for patients in the postoperative period. Important points for clinicians to consider in evaluating the upcoming results will include the relative risk of steroid-induced glaucoma with use of OTX-DP versus conventional topical steroid drops as well as reimbursement practices for insertion of the plug in the operating room following surgery.
More recently, OTX-DP has been investigated for use in the treatment of dry eye [26]. In 2015, a phase 2 clinical trial enrolled 40 patients with dry eye disease. Patients will initially receive a vehicle placebo plug for 30 days, with patients continuing to exhibit symptoms then being randomized to OTX-DP or vehicle placebo plug. Primary outcome measures will include corneal and conjunctival staining, tear breakup time, and resorption of the plug following therapy. Of note, no comparison to conventionally used topical steroid has been done-therefore, it remains unclear whether the low, steady dosing of the OTX-DP is superior to a tapered drop regimen. Further evaluation will be needed to determine whether patients who demonstrate earlier plug resorption will show any decreased efficacy in treatment in either the postoperative or dry eye treatment scenarios and likewise whether the few patients who showed persistence of the plug at 2 months time are at higher risk for adverse events including steroid-induced glaucoma.

\section{EBI 005 (Eleven Biotherapeutics):}

\section{Protein-Based IL-1 Inhibitor}

EBI 005, designed by Eleven Biotherapeutics (Cambridge, MA, USA), is the first protein-based IL-1 inhibitor designed for topical ophthalmic use and has completed phase 2 clinical testing $[27,28]$. IL-1 is an inflammatory mediator of dry eye in addition to multiple systemic diseases. Notably it has been successfully targeted for the treatment of inflammatory conditions such as rheumatoid arthritis. A topically administered ocular therapeutic formulation IL-1 inhibitor was investigated in 2012, and the result, EBI-005, was found to bind its target, IL-1R1, with high specificity, which conferred a high in vivo potency in a therapeutic rat model [27]. 
A phase 2 study was completed in 2015, investigating the use of EBI-005 $5 \mathrm{mg} / \mathrm{ml}$ three times daily for the treatment of allergic conjunctivitis [28]. One hundred fifty-six patients with moderate to severe allergic conjunctivitis were randomized to EBI-005 or vehicle groups and were subjected to aerosolized allergen challenge or direct conjunctival allergen challenge. In the conjunctival allergen challenge test, EBI-005-treated subjects showed statistically significant improvement in ocular itching compared to vehicle. Treated patients also showed greater improvement compared to vehicle in tearing and nasal symptoms. Of note, the primary pre-specified endpoint of ocular itching was not met in the aerosolized challenge test. Further phase 3 clinical testing is under way and will elucidate the role for EBI-005 in both allergic conjunctivitis and dry eye in the clinic.

\section{Diquafosol: $\mathrm{P}_{2} \mathrm{Y}_{2}$ Receptor Agonist}

Diquafosol is a purinergic agonist of the ocular surface $\mathrm{P}_{2} \mathrm{Y}_{2}$ receptor, which promotes fluid transfer and mucin secretion via a pathway involving the activation of phospholipase proteins [29]. Diquafosol was approved in 2010 in Japan for use in treating dry eye, and it recently concluded a phase 3 study in the US [30]. In earlier randomized clinical trials of this medication, mucin production and ocular surface damage appear to be improved, while effects on aqueous production are less certain $[29,31,32]$. There have been no serious ocular side effects reported. Similar to other compounds for treating dry eye, heterogeneity in study design in evaluating clinical endpoints may have contributed to inconsistent findings among the several randomized trials that have been completed for this drug. Further larger trials with longer follow-up periods may better demonstrate its efficacy.

\section{PHARMACEUTICALS IN PHASE 2 CLINICAL TRIALS}

\section{RU-101: Recombinant Human Serum Albumin}

Phase 1 and 2 clinical trials have been completed for RU-101 (R-Tech Ueno, Tokyo, Japan), a topically administered recombinant human serum albumin applied six times daily for the treatment of severe dry eye disease [33]. The phase 1 trial investigated the safety and tolerability of escalating doses of RU-101 over the course of 4 weeks in patients with severe dry eye, and the phase 2 trial investigated the safety and efficacy of the RU-101 dosage established in phase 1 over the course of 12 weeks. The primary outcome measure was safety and secondary outcome measure change from baseline in dry eye symptoms, ocular surface disease index, visual acuity, tear breakup time, corneal fluorescein staining, corneal sensitivity and Schirmer testing [34]. The corneal staining score at 12 weeks was chosen as the primary endpoint for effectiveness. Although there was significant improvement with treatment from baseline to 12 weeks, this was not significantly different from placebo. According to a press release from R-Tech Ueno, plans are underway to further investigate RU-101 in patients with severe dry eye, including dosing optimization. RU-101 or products like it, if effective in clinical trials, could replace human serum tears currently used to treat dry eye, eliminating the 
challenging process of obtaining these medications and avoiding the associated infection risk.

\section{KPI-121/LE-MMP 0.25\%: Loteprednol}

Etabonate Mucus-Penetrating Particle, Glucocorticoid Receptor Agonist

KPI-121 (Kala Pharmaceuticals, Waltham, MA, USA) is a mucus-penetrating nanoparticle loteprednol etabonate $0.25 \%$ topical ophthalmic medication, which has recently completed phase 2 clinical testing in 2015 [35]. The mucus-penetrating particle was designed to enhance delivery into ocular tissues by facilitating tear film mucus penetration [36]. Similar strategies have been previously investigated to deliver medications across the cervical mucosa, gastrointestinal mucosa, and others [37]. The $0.25 \%$ formulation is designed for use in dry eye and meibomian gland disease, whereas a $1 \%$ formulation is targeted for the treatment of postoperative inflammation. The phase 2 trial included 150 patients with dry eye who were randomized to $0.25 \%$ KPI-121 or vehicle placebo, dosed four times daily for 28 days. The primary clinical endpoints were bulbar conjunctival hyperemia and ocular discomfort. There was a statistical difference in conjunctival hyperemia favoring KPI-121, while ocular discomfort showed a favorable trend versus placebo but without a statistical difference. The authors noted that patients with worse baseline ocular discomfort showed a greater trend favoring outcomes with KPI-121 versus placebo. Instillation site pain was reported in $6.9 \%$ of patients receiving the medication versus $3.8 \%$ receiving placebo.

\section{DEVICES IN DEVELOPMENT FOR DRY EYE THERAPY}

\section{Oculeve Neurostimulator Device: Intranasal Lacrimal Stimulator for Dry Eye}

A pilot study has been completed for the Oculeve Neurostim Device (Oculeve, Inc, San Francisco, CA, USA), a lacrimal neural stimulator for the treatment of dry eye [38]. The device, initially conceived by a fellow at the Stanford Biodesign program, is inserted into the mucous membrane in the nasal cavity. Tear production stimulation is then modulated by a wireless controller. The 2015 pilot study, for which results have not yet been published, evaluated the reduction in exacerbation of dry eye symptoms in 40 patients upon exposure to a controlled adverse environment (CAE) in patients with the neurostimulator in place.

\section{Ocular Iontophoresis with EG-437 (40 mg/ ml Dexamethasone Phosphate Solution)}

The EyeGate $^{\circledR}$ II system (Eyegate Pharmaceuticals, Inc, Waltham, MA, USA) is an ocular iontophoriesis system designed to deliver drugs to the conjunctiva and sclera [39, 40]. In ocular iontophoresis, a small current is applied to the ocular surface creating an electrical field, which enhances the mobility of charged particles across the anterior and posterior segments. A drug delivered by ocular iontophoresis may achieve higher concentrations than it would via topical drop form. A recent study of patients with dry eye receiving delivery of EG-437 (40 mg/ml dexamethasone phosphate solution) via ocular iontophoresis found statistically significant 
improvement in signs and symptoms of dry eye in response to a controlled adverse environment challenge. However, the primary endpoints of corneal staining and ocular discomfort were not met in the phase II trial. It should be noted also that $87 \%$ of patients in the safety population had at least one adverse event, most commonly conjunctival hyperemia and keratitis, which required treatment [40]. Some of the patients experiencing adverse events were in the placebo group who received ocular iontophoresis without medication delivery. No severe adverse events were reported. While the clinical results in terms of dry eye endpoints are interesting, future studies and possibly refinements to mitigate the percentage of patients who experience non-serious adverse events with this device may be needed to make it a success in the clinical setting.

\section{CONCLUSION}

We are at an exciting crossroads with respect to dry eye treatment. Multiple pharmaceutical agents are moving toward the market for the treatment of dry eye syndrome. Topical agents range from twice daily to four times daily administration, and phase 3 trials have shown varied degrees of improvement in subjective and objective signs of dry eye syndrome. One important remaining question is, given the chronic nature of the disease, how well will these medications be tolerated when taken over a long time period and will their effectiveness remain the same when taken chronically? In addition to studies of potential long-term local side effects, whether there are any systemic side effects of the immunomodulatory medications remains an important question, despite their low levels of systemic absorption. This remains to be shown through longer-term studies following phase 3 clinical trials.

Inflammation is a common feature in multiple ocular surface diseases in addition to dry eye, including blepharitis, allergic conjunctivitis, and others. Thus, agents designed to treat dry eye may have cross-over benefit to those suffering from other ocular surface conditions, and vice versa. Further study of some of these medications for patients with other inflammatory diseases is merited and in some cases is already underway. Patients with more than one inflammatory ocular surface disease (as is often the case) represent an important cohort to study as well. The future is bright as medications and devices continue to flow into the pipeline. Dry eye sufferers and physicians alike can only hope that in the decade to come we will have many more approved therapeutics available to treat dry eye.

\section{ACKNOWLEDGMENTS}

No funding or sponsorship was received for this study or publication of this article.

All named authors meet the International Committee of Medical Journal Editors (ICMJE) criteria for authorship for this manuscript, take responsibility for the integrity of the work as a whole, and have given final approval for the version to be published.

Conflict of interest. P. K. Gupta is a consultant for AMO, Shire, Allergan, Tear Science, Novabay, and BioTissue, all of which have products related to the treatment of dry eye. L. A. Vickers declares no conflict of interest.

Compliance with ethics guidelines. This article is based on previously conducted studies and does not involve any new studies 
of human or animal subjects performed by any of the authors.

Open Access. This article is distributed under the terms of the Creative Commons Attribution-NonCommercial 4.0 International License (http://creativecommons.org/licenses/ by-nc/4.0/), which permits any noncommercial use, distribution, and reproduction in any medium, provided you give appropriate credit to the original author(s) and the source, provide a link to the Creative Commons license, and indicate if changes were made.

\section{REFERENCES}

1. Epidemiology Subcommittee DEWS Workshop. The epidemiology of dry eye disease: report of the Epidemiology Subcommittee of the International Dry Eye WorkShop (2007). Ocul Surf. 2007;5(2):93-107.

2. Raoof D, Pineda R. Dry eye after laser in situ keratomileusis. Semin Ophthalmol. 2014;29(5-6):358-62.

3. Brown MM, Brown GC, Brown $\mathrm{HC}$, et al. Value-based medicine, comparative effectiveness, and cost-effectiveness analysis of topical cyclosporine for the treatment of dry eye syndrome. Arch Ophthalmol. 2009;127(2):146-52.

4. Schein OD, Hochberg MC, Munoz B, et al. Dry eye and dry mouth in the elderly: a population-based assessment. Arch Intern Med. 1999;159(12):1359-63.

5. Moss SE, Klein R, Klein BE. Prevalence of and risk factors for dry eye syndrome. Arch Ophthalmol. 2000;118(9):1264-8.

6. Munoz B, West SK, Rubin GS, et al. Causes of blindness and visual impairment in a population of older Americans: the Salisbury Eye Evaluation Study. Arch Ophthalmol. 2000;118(6):819-25.

7. Sheppard JD, Torkildsen GL, Lonsdale JD, et al. Lifitegrast ophthalmic solution $5.0 \%$ for treatment of dry eye disease: results of the OPUS-1 phase 3 study. Ophthalmology. 2014;121(2):475-83.

8. Yandrapu S, Kompella UB. Development of sustained-release microspheres for the delivery of SAR 1118, an LFA-1 antagonist intended for the treatment of vascular complications of the eye. J Ocul Pharmacol Ther. 2013;29(2):236-48.
9. Paskowitz DM, Nguyen QD, Gehlbach P, et al. Safety, tolerability, and bioavailability of topical SAR 1118, a novel antagonist of lymphocyte function-associated antigen-1: a phase $1 \mathrm{~b}$ study. Eye (Lond). 2012;26(7):944-9.

10. http://www.prnewswire.com/news-releases/shirereports-top-line-results-on-opus-2-a-phase-3-studyinvestigating-the-use-of-lifitegrast-50-ophthalmicsolution-in-adults-with-dry-eye-disease-234660341. html.

11. http://www.medscape.com/viewarticle/843968.

12. Kashima $\mathrm{T}$, Itakura $\mathrm{H}$, Akiyama $\mathrm{H}$, Kishi $\mathrm{S}$. Rebamipide ophthalmic suspension for the treatment of dry eye syndrome: a critical appraisal. Clin Ophthalmol. 2014;8:1003-10.

13. Kinoshita S, Awamura S, Oshiden $\mathrm{K}$, et al. Rebamipide (OPC-12759) in the treatment of dry eye: a randomized, double-masked, multicenter, placebo-controlled phase II study. Ophthalmology. 2012;119(12):2471-8.

14. Koh $\mathrm{S}$, Inoue $\mathrm{Y}$, Sugmimoto $\mathrm{T}$, et al. Effect of rebamipide ophthalmic suspension on optical quality in the short break-up time type of dry eye. Cornea. 2013;32(9):1219-23.

15. Igarashi A, Kamiya K, Kobashi H, Shimizu K. Effect of rebamipide ophthalmic suspension on intraocular light scattering for dry eye after corneal refractive surgery. Cornea. 2015;34(8):895-900.

16. Kinoshita S, Oshiden $\mathrm{K}$, Awamura $\mathrm{S}$, et al. A randomized, multicenter phase 3 study comparing $2 \%$ rebamipide (OPC-12759) with $0.1 \%$ sodium hyaluronate in the treatment of dry eye. Ophthalmology. 2013;120(6):1158-65.

17. Takeji Y, Urashima $H$, Aoki A, Shinohara $H$. Rebamipide increases the mucin-like glycoprotein production in corneal epithelial cells. J Ocul Pharmacol Ther. 2012;28(3):259-63.

18. Urashima $\mathrm{H}$, Okamoto $\mathrm{T}$, Takeji $\mathrm{Y}$, et al. Rebamipide increases the amount of mucin-like substances on the conjunctiva and cornea in the $\mathrm{N}$-acetylcysteine-treated in vivo model. Cornea. 2004;23(6):613-9.

19. Meerovitch K, Torkildsen G, Lonsdale J, et al. Safety and efficacy of MIM-D3 ophthalmic solutions in a randomized, placebo-controlled Phase 2 clinical trial in patients with dry eye. Clin Ophthalmol. 2013;7:1275-85.

20. Jain P, Li R, Lama $\mathrm{T}$, et al. An NGF mimetic, MIM-D3, stimulates conjunctival cell glycoconjugate secretion and demonstrates 
therapeutic efficacy in a rat model of dry eye. Exp Eye Res. 2011;93(4):503-12.

21. https://clinicaltrials.gov/ct2/show/NCT01960010.

22. http://www.mimetogen.com/clinical-development. html.

23. http://www.mimetogen.com/news-publications/ press-releases/51-mimetogen-pharmaceuticalsannounces-topline-results-of-its-second-clinicalstudy-with-mim-d3-for-the-treatment-of-dryeye-syndrome.html.

24. http://investors.ocutx.com/phoenix.zhtml?c=2536 50\&p=irol-newsArticle\&ID=2032479.

25. http://www.ocutx.com/pipeline/dexamethasonepunctum-plug.

26. http://investors.ocutx.com/phoenix.zhtml?c=253650 $\& p=$ irol-newsArticle\&ID=2009132.

27. Hou J, Townson SA, Kovalchin JT, et al. Design of a superior cytokine antagonist for topical ophthalmic use. Proc Natl Acad Sci. 2013;110(10):3913-8.

28. Goldstein $\mathrm{MH}$, Tubridy $\mathrm{KL}$, Agahigian J, et al. A phase 2 exploratory study of a novel interleukin-1 receptor inhibitor (EBI-005) in the treatment of moderate-to-severe allergic conjunctivitis. Eye Contact Lens. 2015;41(3):145-55.

29. Keating GM. Diquafosol ophthalmic solution 3\%: a review of its use in dry eye. Drugs. 2015;75(8):911-22.

30. https://clinicaltrials.gov/ct2/show/NCT00831662.

31. Matsumoto Y, Ohashi Y, Watanabe H, et al. Efficacy and safety of diquafosol ophthalmic solution in patients with dry eye syndrome: a Japanese phase 2 clinical trial. Ophthalmology. 2012; 119(10):1954-60.
32. Takamura E, Tsubota $\mathrm{K}$, Watanabe $\mathrm{H}$, et al. A randomised, double-masked comparison study of diquafosol versus sodium hyaluronate ophthalmic solutions in dry eye patients. Br J Ophthalmol. 2012;96(10):1310-5.

33. https://clinicaltrials.gov/ct2/show/NCT01843894.

34. http://www.businesswire.com/news/home/201411 09005056/en/R-Tech-Ueno-Completion-Phase-IIIClinical-Study-.VYOo6mAXN6k.

35. Schopf L, Enlow E, Popov A, et al. Ocular pharmacokinetics of a novel loteprednol etabonate $\quad 0.4 \%$ ophthalmic formulation. Ophthalmol Ther. 2014. doi:10.1007/s40123-0140021-z.

36. http://www.businesswire.com/news/home/201504 01005554/en/Kala-Pharmaceuticals-AnnouncesPositive-Results-Phase-2-.VYOvQWAXN6k.

37. Lai SK, O'Hanlon DE, Harrold S, et al. Rapid transport of large polymeric nanoparticles in fresh undiluted human mucus. Proc Natl Acad Sci. 2007;104(5):1482-7.

38. https://clinicaltrials.gov/ct2/show/NCT02313454.

39. Gungor S, Delgado-Charro MB, Ruiz-Perez B, et al. Trans-scleral iontophoretic delivery of low molecular weight therapeutics. J Control Release. 2010;147(2):225-31.

40. Patane MA, Cohen A, From S, et al. Ocular iontophoresis of EGP-437 (dexamethasone phosphate) in dry eye patients: results of a randomized clinical trial. Clin Ophthalmol. 2011;5:633-43. 